\title{
Downregulation of MiR-199b-5p is Associated With Chemoresistance Via Jagged1 Mediated Notch Signaling in Human Ovarian Cancer Cells
}

X. Liu, W. Chan, Y.S. Ngan. 1Faculty of Medicine, Obstetrics and Gynaecology, Pokfulam, Hong Kong

BACKGROUND: Ovarian cancer is one of the most deadly gynaecological malignancies worldwide. Adjuvant chemotherapy combined with other treatments is the current management of the advanced ovarian cancers. However, the acquisition of resistance to initially responsive tumors limits the successful curative rate. The underlying molecular mechanism remains largely unknown. Here, we report that the downregulation of miR199b-5p leading to elevated JAG1-mediated NOTCH signaling activity may be involved in chemoresistance of human ovarian cancer cells.

MATERIAL AND METHODS: MicroRNA199b-5p (miR-199b-5p) expression was determined by Exiqon LNA ${ }^{\mathrm{TM}}$ miRNA microarray and quantitative RT-PCR (QPCR) analyses. The demethylation treatments using 5-Aza-dC and/or TSA were applied to show promoter hypermethylating scenario of miR-199b-5p in ovarian cell lines. Cell growth was assessed by XTT cell viability and colony formation assays. The computational softwares prediction, 3_UTR luciferase reporter assay and western blotting were used to validate targets of miR-199b-5p. The g-secretase inhibitor was used to inhibit Notch activity in ovarian cancer cells. Commercial siRNA and miRNA inhibitor were used for knockdown JAG1 and miR-199b-5p respectively.

RESULTS: Using Exiqon LNA ${ }^{\mathrm{TM}}$ miRNA microarray profiling and Q-PCR analyses, we found that miR-199b-5p was frequently downregulated in cisplatin-resistant cell lines and was significantly associated with advanced stage $(P=0.015)$ and high grade $(P=0.003)$ ovarian cancer samples $(\mathrm{N}=31)$. Intriguingly, the expression of miR-199b-5p could be restored upon treatments of 5-Aza-dC and TSA in ovarian cancer cell lines $(\mathrm{N}=5)$. Functionally, re-expression of miR-199b-5p remarkably diminished cisplatin-resistance, wheareas depletion of miR-199b-5p by siRNA approach augmented cisplatin resistance in ovarian cancer cells. Computational prediction and western blotting identified JAG1, a significant Notch signaling effector, as a putative target of miR-199b-5p. Luciferase reporter assay using wild type or mutated miR-199b-5p targeting site at 3_UTR of JAG1 further demonstrated that miR-199b-5p could specifically repress JAG1 expression dose dependenly. Importantly, enforced expression of JAG1 increased not only Notch signaling activity but also cisplatin-resistance in ovarian cancer cells. In contrast, knockdown of JAG1 by siRNA impaired the cisplatin-resistance of ovarian cancer cells. Furthermore, inhibition of Notch signaling by g-secretase inhibitor showed the same effect in sensitizing ovarian cancer cells to cisplatin induced cell apoptosis, indicating that Notch signaling is involved in chemoresistance of ovarian cancer.

CONCLUSION: Our data suggest that the aberrant activation of JAG1/Notch signaling due to epigenetic silencing of miR-199b-5p may contribute to chemoresistance in human ovarian cancer cells. 